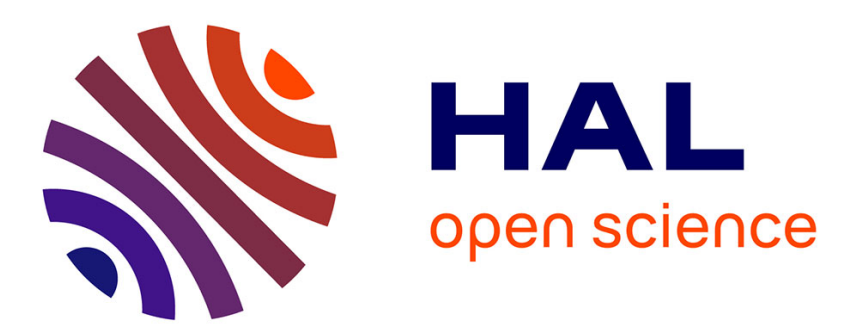

\title{
The preferred mode of incompressible jets: linear frequency response analysis
}

Xavier Garnaud, Lutz Lesshafft, Peter Schmid, Patrick Huerre

\section{To cite this version:}

Xavier Garnaud, Lutz Lesshafft, Peter Schmid, Patrick Huerre. The preferred mode of incompressible jets: linear frequency response analysis. Journal of Fluid Mechanics, 2013, 716, pp. 189-202. $10.1017 / \mathrm{jfm} .2012 .540$. hal-00756811

\section{HAL Id: hal-00756811 https://hal.science/hal-00756811}

Submitted on 23 Nov 2012

HAL is a multi-disciplinary open access archive for the deposit and dissemination of scientific research documents, whether they are published or not. The documents may come from teaching and research institutions in France or abroad, or from public or private research centers.
L'archive ouverte pluridisciplinaire HAL, est destinée au dépôt et à la diffusion de documents scientifiques de niveau recherche, publiés ou non, émanant des établissements d'enseignement et de recherche français ou étrangers, des laboratoires publics ou privés. 


\title{
The preferred mode of incompressible jets: linear frequency response analysis
}

\author{
X. GARNA UD, L. LESSHAFFT, P. J. SCHM ID \\ AND P. HUERRE \\ Laboratoire d'Hydrodynamique (LadHyX), Ecole Polytechnique - CNRS, 91128 Palaiseau, \\ France
}

(Received 23 November 2012)

The linear amplification of axisymmetric external forcing in incompressible jet flows is investigated within a fully non-parallel framework. Experimental and numerical studies have shown that isothermal jets preferably amplify external perturbations for Strouhal numbers in the range $0.25 \leq S t_{D} \leq 0.5$, depending on the operating conditions. In the present study, the optimal forcing of an incompressible jet is computed as a function of the excitation frequency. This analysis characterizes the preferred amplification as a pseudo-resonance with a dominant Strouhal number of around 0.45. The flow response at this frequency takes the form of a vortical wavepacket that peaks inside the potential core. Its global structure is characterized by the cooperation of local shear-layer and jet-column modes.

\section{Introduction}

Large-scale coherent structures develop in the shear-layers of isothermal jet flows, for both laminar and turbulent regimes. These structures are not self-sustained, but are the consequence of strong amplification of incoming disturbances. Crow \& Champagne (1971) performed experiments where the flow was forced with a controlled frequency; they showed that optimal excitation is achieved for a Strouhal number based on the jet diameter of about 0.3. The corresponding flow perturbations, referred to as the jet preferred mode, grow in amplitude starting at the nozzle until they undergo non-linear saturation.

A local analysis of jets (Michalke 1984) identifies shear-layer perturbations immediately downstream of the nozzle as the fastest growing instability modes, which would indicate that the preferred frequency scales with the initial shear-layer thickness. This also suggests that the mechanisms underlying the selection of the preferred frequency depend on the downstream flow development. Under the assumption of a slowly diverging base flow, Crighton \& Gaster (1976) used a WKBJ approximation to describe the spatial development of the instability wave. Their results are in reasonable agreement with the experimental results of Crow \& Champagne (1971) for the initial growth of the structures. This approach has subsequently been generalized through the use of the parabolized stability equations (Ray et al. 2009; Gudmundsson \& Colonius 2011; Rodriguez et al. 2011). While this approach also relies on the assumption of a slow variation of the base flow in the streamwise direction, these results were found to yield good agreement with experiments of natural turbulent jets.

The optimal disturbance of flows subjected to time-harmonic linear perturbations has been described by Trefethen et al. (1993). This method was first applied to general non-parallel configurations using a projection of the flow dynamics onto a reduced space spanned by a set of eigenmodes (Alizard et al. 2009; Nichols \& Lele 2010). In other studies 
(Monokrousos et al. 2010; Marquet \& Sipp 2010; Nichols \& Lele 2011b; Sipp \& Marquet 2012) the resolvent norm has been computed directly from the linearized Navier-Stokes operator, such that the entire non-normal flow behavior captured by the discretization is taken into account in the results. This approach is followed here to provide a better understanding of the preferred frequency selection and the associated spatial structures; in particular, the non-parallel nature of the flow, as well as the effects of a solid circular jet-pipe, are taken into account. The present analysis is mainly restricted to axisymmetric forcing and perturbations. Results for helical forcing are only briefly discussed.

After a description of the flow under consideration in $\S 2$, two different models of the external forcing are described in $\S 4$, together with the numerical method used. The results, presented in $\S 5$, are then discussed and compared to classical local stability analysis.

\section{Flow configuration}

\subsection{Geometry}

A cylindrical jet of an incompressible Newtonian fluid of viscosity $\nu^{*}$, with radius $R^{*}$ and exit centerline velocity $U_{0}^{*}$ is considered. The latter two quantities are used to make lengths and velocities non-dimensional. Frequencies $f^{*}$ can be non-dimensionalized to yield either a circular frequency $\omega$ or a Strouhal number $S t$ based on the jet diameter. These parameters are related via $S t=\omega / \pi$. Throughout the study, the Reynolds number is taken to be

$$
R e=\frac{U_{0}^{*} R^{*}}{\nu^{*}}=10^{3} .
$$

The flow geometry, described in terms of the cylindrical coordinates $r, \theta$ and $x$, is represented in figure 1 . The boundary of the computational domain $\Omega$ is decomposed into $\Gamma_{i}, \Gamma_{w}, \Gamma_{o}$ and $\Gamma_{a}$ respectively corresponding to the inlet, a solid wall, the outlet and the jet axis. No-slip boundary conditions are imposed on $\Gamma_{w}$, and stress-free boundary conditions are used on $\Gamma_{o}$ (Dick 2009). Compatibility conditions, ensuring the smoothness of the computed fields are imposed on the axis $r=0$ (Matsushima \& Marcus 1995). At the inflow, homogeneous or inhomogeneous Dirichlet boundary conditions are imposed on the velocity as requested by the problem under consideration.

Two unstructured meshes with identical dimensions but different resolution are used for the finite element computations. The density of vertices in the domain is controlled by the distance between discretization points on the boundary of the computational domain as well as on interior boundaries (dashed lines in figure 1). This distance is denoted by $h_{4}$ for boundaries in the far field $\left(r>r_{3}^{+}\right)$. It is smaller than $h_{3}$ for $r \leq r_{3}^{+}$, and respectively smaller than $h_{2}$ and $h_{1}$ in the inner regions defined by $x \leq x_{2}^{+}$and $1-\delta_{2} / 2 \leq r \leq 1+\delta_{2} / 2$ and by $x_{1}^{-} \leq x \leq x_{1}^{+}$and $1-\delta_{1} / 2 \leq r \leq 1+\delta_{1} / 2$. These subdomains are indicated by gray shaded areas in figure 1 . The values of the $h_{i}$ for both meshes are given in figure 1 .

\subsection{Base state}

Linear stability analysis formally applies to base states that are steady solutions of the governing equations. However, several studies have found that linearization around a time-averaged mean flow yields better predictions of the nonlinear flow behaviour, in particular with regard to the frequency selection of intrinsic oscillations (Pier 2002; Barkley 2006). The present study employs the mean-flow model proposed by Monkewitz \& Sohn (1988) for a turbulent free jet, displayed in figure 2. This model consists of a potential core, starting from a momentum thickness $\theta=0.043$, and extending over eight radii 


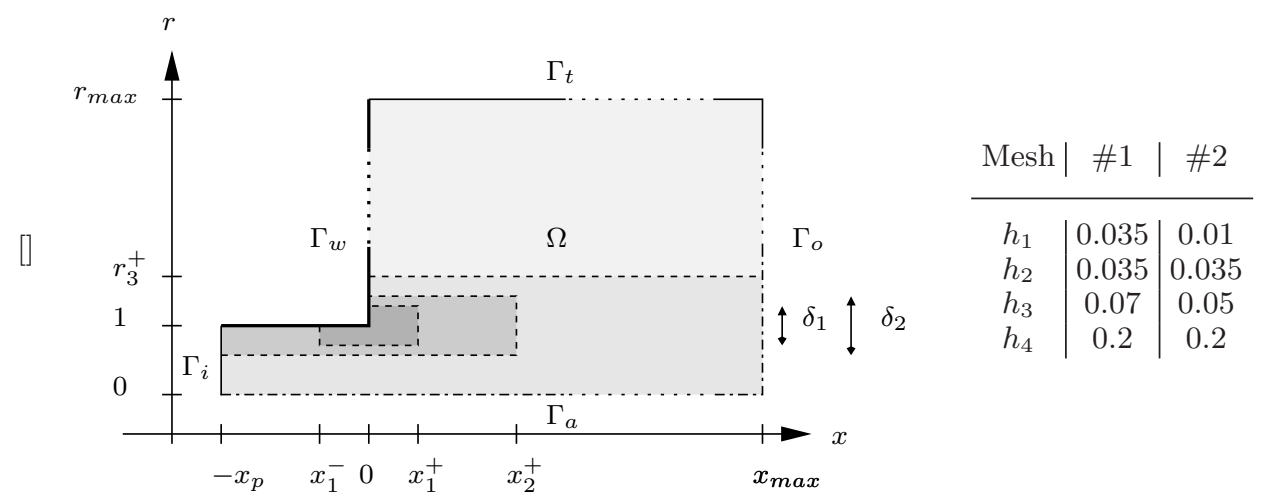

FIGURE 1. Schematic representation of the numerical domain. The pipe length is set to $x_{p}=5$, and it has been verified that setting $r_{\max }=10$ does not influence the results. Gray shaded areas correspond to subregions in which different grid resolutions are selected. Values of $x_{1}^{-}=-2$, $x_{1}^{+}=1, \delta_{1}=0.15, x_{2}^{+}=10, \delta_{2}=0.5$ and $r_{3}^{+}=2$ are used.

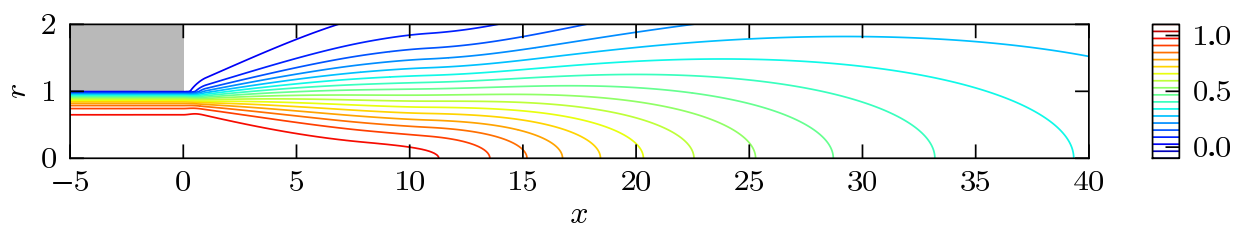

Figure 2. Axial velocity field for the model turbulent mean flow of Monkewitz \& Sohn (1988).

downstream of the end of the jet pipe, followed by a self-similar region where the velocity profiles have an approximately Gaussian shape. A parallel pipe flow region has been added upstream, and a smooth transition is made for $0 \leq x \leq 1$, as described in Garnaud et al. (2011).

In the following, infinitesimal perturbations around the steady mean flow are considered, such that the flow field can be written as $(\boldsymbol{u}, p)=\left(\boldsymbol{U}+\epsilon \boldsymbol{u}^{\prime}, P+\epsilon p^{\prime}\right)$, where $(\boldsymbol{U}, P)$ denotes the base state displayed in figure 2 .

\section{Modal analyis}

Monkewitz (1989) and Huerre \& Monkewitz (1990) conjectured that the preferred mode observed in experiments corresponds to the resonance of the least stable eigenmode of the jet with incoming disturbances. This issue has been investigated by Cooper \& Crighton (2000) by means of a WKBJ approximation. Upon making the assumptions that (i) the global mode has the shape of a local shear-layer mode at each location, and that (ii) the base flow development is slow, the authors found a weakly stable global mode at a Strouhal number of 0.44 which agrees well with experimental observations. In order to avoid such strong assumptions, eigenmodes can now be computed using the axisymmetric Navier-Stokes equations discretized on a two-dimensional domain. Such a modal analysis has for example been performed by Nichols \& Lele (2011a) in the context of supersonic jets. This approach is followed in this section. Figure 3 displays the 


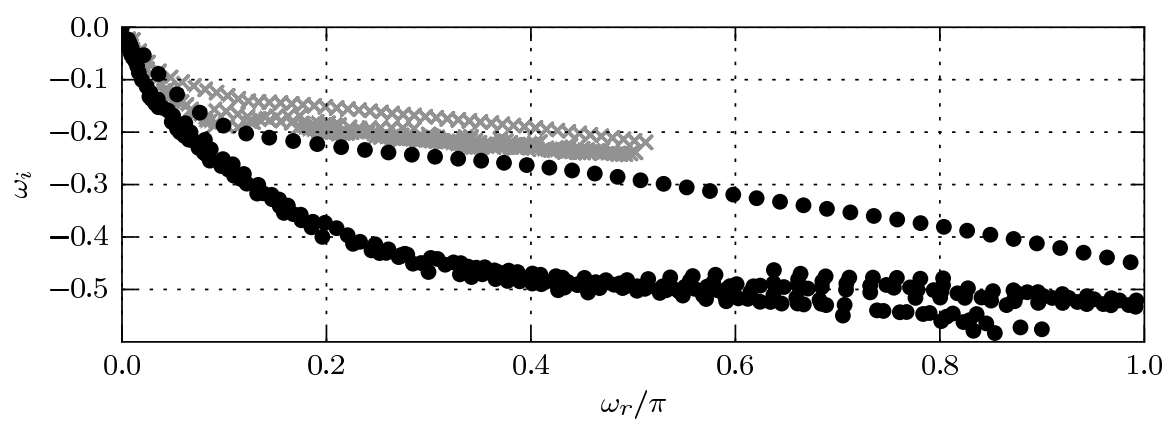

FiguRE 3. Eigenvalue spectra of the linearized Navier-Stokes equations ( $L \boldsymbol{q}=\mathrm{i} \omega B \boldsymbol{q})$ computed for a domain of length $x_{\max }=40$ (black dots) and $x_{\max }=60$ (gray crosses).

spectrum obtained for the global eigenvalue problem

$$
\begin{aligned}
\boldsymbol{\nabla} \cdot \boldsymbol{u}^{\prime} & =0, \\
-\mathrm{i} \omega \boldsymbol{u}^{\prime}+(\boldsymbol{U} \cdot \boldsymbol{\nabla}) \boldsymbol{u}^{\prime}+\left(\boldsymbol{u}^{\prime} \cdot \boldsymbol{\nabla}\right) \boldsymbol{U} & =-\boldsymbol{\nabla} p^{\prime}+\frac{1}{R e} \nabla^{2} \boldsymbol{u}^{\prime}+\psi(\boldsymbol{x}) \boldsymbol{f}^{\prime},
\end{aligned}
$$

with homogeneous Dirichlet boundary conditions on $\Gamma_{i} \cup \Gamma_{w}$. All eigenmodes are stable, and three families of modes can be identified. First, low-frequency free-stream modes (eigenvalues close to the origin) correspond to standing vortical structures. These decay very slowly due to viscous effects. Second, a branch of shear-layer / jet-column modes is observed (upper branch in figure 3). Along this branch the decay rate $-\omega_{i}$ increases with frequency $\omega_{r}$, and the spatial structure of the eigenmodes is characterized by an exponential growth throughout the computational domain. This growth can be understood as a consequence of the stable advection of nearly neutral shear-layer structures. Finally, the lower branch of eigenmodes in figure 3 corresponds in fact to pseudomodes that lie on the $10^{-10}$ contour of the pseudospectrum. Note that the actual spectrum is quite dependent on the size of the numerical domain, but that qualitative features are not. For more details, see Garnaud (2012).

The spectrum of the linearized Navier-Stokes equations therefore exhibits no isolated or least stable eigenmode that could explain the preferred mode through a resonance mechanism. In the next section, a pseudo-resonance analysis is carried out to investigate the origin of the preferred mode.

\section{Response to harmonic forcing}

\subsection{External forcing as a body force}

Following Monokrousos et al. (2010), Marquet \& Sipp (2010) and Sipp \& Marquet (2012), the external forcing can be modeled as a body force $\boldsymbol{f}(\boldsymbol{x}, t)$ acting on the momentum equation,

$$
\frac{\partial \boldsymbol{u}}{\partial t}+(\boldsymbol{u} \cdot \boldsymbol{\nabla}) \boldsymbol{u}=-\boldsymbol{\nabla} p+\frac{1}{R e} \boldsymbol{\nabla}^{2} \boldsymbol{u}+\psi(\boldsymbol{x}) \boldsymbol{f},
$$

while $\boldsymbol{\nabla} \cdot \boldsymbol{u}=0$ is maintained throughout the flow. The weight function $\psi$ is used to restrict the flow region where forcing is applied, and the forcing amplitude is assumed to be small: $\boldsymbol{f}=\epsilon \boldsymbol{f}^{\prime}$. To leading order, the dynamics of perturbations are governed by the 
linear system

$$
\begin{array}{rlrl}
\nabla \cdot \boldsymbol{u}^{\prime} & =0, & & \\
\frac{\partial \boldsymbol{u}^{\prime}}{\partial t}+(\boldsymbol{U} \cdot \nabla) \boldsymbol{u}^{\prime}+\left(\boldsymbol{u}^{\prime} \cdot \nabla\right) \boldsymbol{U} & =-\nabla p^{\prime}+\frac{1}{R e} \nabla^{2} \boldsymbol{u}^{\prime}+\psi(\boldsymbol{x}) \boldsymbol{f}^{\prime}, & & \\
\boldsymbol{u}^{\prime} & =0, & \Gamma_{i} \cup \Gamma_{w} \\
\frac{1}{R e} \frac{\partial \boldsymbol{u}^{\prime}}{\partial n}-p^{\prime} \boldsymbol{n} & =0 . & \Gamma_{t} \cup \Gamma_{o}
\end{array}
$$

In a linear framework, all signals are decomposed in time into independent Fourier components. The forcing is therefore considered to be time-harmonic, $\boldsymbol{f}^{\prime}=\widetilde{\boldsymbol{f}} \exp (-\mathrm{i} \omega t)$, prompting an asymptotic flow response $\left(\boldsymbol{u}^{\prime}, p^{\prime}\right)=(\widetilde{\boldsymbol{u}}, \widetilde{p}) \exp (-\mathrm{i} \omega t)$ at the same frequency. The amplification of the externally applied forcing at a given frequency $\omega$ is measured in terms of the gain

$$
G_{o p t}^{b f}(\omega)=\max _{\widetilde{\boldsymbol{f}}}\left(\int_{\Omega}|\widetilde{\boldsymbol{u}}|^{2} r \mathrm{~d} r \mathrm{~d} x\right) /\left(\int_{\Omega}|\widetilde{\boldsymbol{f}}|^{2} r \mathrm{~d} r \mathrm{~d} x\right) .
$$

The optimal forcing $\widetilde{\boldsymbol{f}}_{\text {opt }}(\omega)$ realizes this maximum. For the results presented in $\S 5.1$, the forcing is assumed to be localized inside the pipe only, i.e. the weight function is defined as $\psi(\boldsymbol{x})=1$ for $x<0$ and $\psi(\boldsymbol{x})=0$ for $x \geq 0$.

\subsection{External forcing as an inflow condition}

Rather than forcing the jet through a distributed body force in the pipe interior as in the previous section, one may model incoming perturbations in the form of an unsteady upstream boundary condition of the linearized Navier-Stokes equations:

$$
\begin{array}{rlrl}
\nabla \cdot \boldsymbol{u}^{\prime} & =0, & & \\
\frac{\partial \boldsymbol{u}^{\prime}}{\partial t}+(\boldsymbol{U} \cdot \nabla) \boldsymbol{u}^{\prime}+\left(\boldsymbol{u}^{\prime} \cdot \nabla\right) \boldsymbol{U} & =-\nabla p^{\prime}+\frac{1}{R e} \nabla^{2} \boldsymbol{u}^{\prime}, & & \\
\boldsymbol{u}^{\prime} & =0, & \Gamma_{w} \\
\frac{1}{R e} \frac{\partial \boldsymbol{u}^{\prime}}{\partial n}-p^{\prime} \boldsymbol{n} & =0, & \Gamma_{t} \cup \Gamma_{o}
\end{array}
$$

Such a model corresponds more closely to the assumptions of local spatial stability, WKBJ and PSE approximations. In this case, the gain between a harmonic inflow forcing and the corresponding response is measured as

$$
G_{o p t}^{b c}(\omega)=\max _{\widetilde{\boldsymbol{f}}}\left(\int_{\Omega}|\widetilde{\boldsymbol{u}}|^{2} r \mathrm{~d} r \mathrm{~d} x\right) /\left(\int_{\Gamma_{i}}|\widetilde{\boldsymbol{f}}|^{2} r \mathrm{~d} r\right) .
$$

\subsection{Numerical solution of the optimization problem}

The linear systems (4.1) and (4.3) are discretized by $P 2-P 1$ finite element using the software FreeFEM++ (Hecht 2011). Let $\boldsymbol{q}$ be the discrete state vector containing all degrees of freedom related to velocity and pressure fields. Both (4.1) and (4.3) can then be written in their semi-discretized form as

$$
B \dot{\boldsymbol{q}}=L \boldsymbol{q}+B_{f} \boldsymbol{f},
$$

where $f$ is the discrete forcing vector and $L, B$ and $B_{f}$ are sparse matrices resulting from the finite elements discretization of the linearized Navier-Stokes equations. Let 
$\boldsymbol{f}=\tilde{\boldsymbol{f}} \exp (-\mathrm{i} \omega t)$ and $\boldsymbol{q}=\widetilde{\boldsymbol{q}} \exp (-\mathrm{i} \omega t)$ be time-harmonic such that

$$
-(L+\mathrm{i} \omega B) \widetilde{\boldsymbol{q}}=B_{f} \widetilde{\boldsymbol{f}} .
$$

Perturbation amplitudes are measured in a pseudonorm $\|\boldsymbol{q}\|^{2}=\boldsymbol{q}^{\dagger} Q \boldsymbol{q}$ that represents the discretization of the perturbation kinetic energy:

$$
\left\|\left(\boldsymbol{u}^{\prime}, p^{\prime}\right)\right\|^{2}=\int_{\Omega}\left|\boldsymbol{u}^{\prime}\right|^{2} r \mathrm{~d} r \mathrm{~d} x .
$$

The norm of the forcing vector $\boldsymbol{f}$, which appears in the denominator of (4.2) and (4.4), is expressed accordingly in discrete form as $\|\boldsymbol{f}\|_{f}^{2}=\boldsymbol{f}^{\dagger} Q_{f} \boldsymbol{f}$. Note that $\boldsymbol{f}$ does not contain pressure components, and that $Q_{f}$ therefore is symmetric positive-definite, in contrast to $Q$, which is positive semi-definite. The discrete optimal forcing problem can be written as

$$
G_{o p t}^{2}(\omega)=\max \frac{\|\widetilde{\boldsymbol{q}}\|^{2}}{\|\widetilde{\boldsymbol{f}}\|_{f}^{2}} .
$$

Monokrousos et al. (2010) formalized a similar optimal forcing problem by use of a constrained optimization approach involving Lagrange multipliers. For linear timeharmonic problems, a more concise formalism is possible. The formulation used here, similar to that of Sipp \& Marquet (2012), is briefly outlined below. Substituting (4.6) into (4.8) gives

$$
\begin{aligned}
G_{\text {opt }}(\omega)^{2} & =\max _{\widetilde{\boldsymbol{f}}} \frac{\left\|(L+\mathrm{i} \omega B)^{-1} B_{f} \widetilde{\boldsymbol{f}}\right\|^{2}}{\|\widetilde{\boldsymbol{f}}\|_{f}^{2}}, \\
& =\max _{\widetilde{\boldsymbol{f}}} \frac{\widetilde{\boldsymbol{f}}^{\dagger} B_{f}^{\dagger}(L+\mathrm{i} \omega B)^{-1^{\dagger}} Q^{\dagger}(L+\mathrm{i} \omega B)^{-1} B_{f} \widetilde{\boldsymbol{f}}}{\widetilde{\boldsymbol{f}}^{\dagger} Q_{f} \widetilde{\boldsymbol{f}}} .
\end{aligned}
$$

Let $M_{f}^{\dagger} M_{f}$ be the Cholesky decomposition of $Q_{f}$, and let $\widetilde{\boldsymbol{g}}=M_{f} \widetilde{\boldsymbol{f}}$, i.e. $\widetilde{\boldsymbol{f}}=M_{f}^{-1} \widetilde{\boldsymbol{g}}$. The optimal gain can then be rewritten as

$$
G_{o p t}(\omega)^{2}=\max _{\widetilde{\boldsymbol{g}}} \frac{\widetilde{\boldsymbol{g}} M_{f}^{-1^{\dagger}} B_{f}^{\dagger}(L+\mathrm{i} \omega B)^{-1^{\dagger}} Q^{\dagger}(L+\mathrm{i} \omega B)^{-1} B_{f} M_{f}^{-1} \widetilde{\boldsymbol{g}}}{\widetilde{\boldsymbol{g}}^{\dagger} \widetilde{\boldsymbol{g}}} .
$$

The right-hand side of the above expression is a Rayleigh quotient, and $G_{o p t}(\omega)$ is therefore the leading eigenvalue of the associated Hermitian eigenvalue problem

$$
M_{f}^{-1^{\dagger}} B_{f}^{\dagger}(L+\mathrm{i} \omega B)^{-1^{\dagger}} Q^{\dagger}(L+\mathrm{i} \omega B)^{-1} B_{f} M_{f}^{-1} \widetilde{\boldsymbol{g}}=\lambda \widetilde{\boldsymbol{g}},
$$

which can be re-written in terms of the forcing $\widetilde{\boldsymbol{f}}$ as

$$
Q_{f}^{-1} B_{f}^{\dagger}(L+\mathrm{i} \omega B)^{-1 \dagger} Q^{\dagger}(L+\mathrm{i} \omega B)^{-1} B_{f} \tilde{\boldsymbol{f}}=\lambda \tilde{\boldsymbol{f}} .
$$

The leading eigenvalue of (4.9) and its associated eigenvector, which respectively correspond to the optimal gain and optimal forcing, are computed by using the Lanczos solver implemented in SLEPc (Hernandez et al. 2005). The operator $(L+\mathrm{i} \omega B)^{-1}$ and its adjoint are applied by using the sparse linear algebra package MUMPS through its PETSc interface (Balay et al. 2008). Finally, the operator $Q_{f}^{-1}$ is applied by using a Cholesky decomposition, if memory requirements permit, or otherwise by using an ILUpreconditioned conjugate gradient method. 

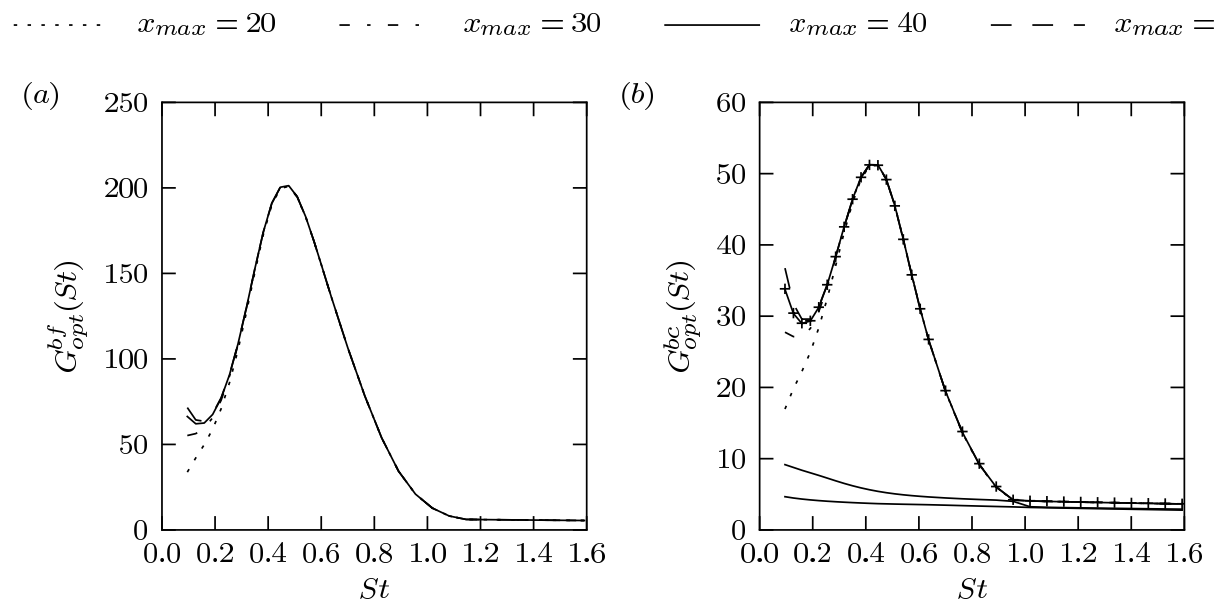

FiguRE 4. Optimal gain as a function of the Strouhal number for body $(a)$ and boundary $(b)$ forcing. The gains are computed for various domain lengths $x_{\max } .+$ symbols displayed in $(b)$ correspond to gains computed for a finer mesh (mesh \#2 in figure 1), showing convergence with respect to grid resolution. For boundary forcing on a domain of length $x_{\max }=40$, not only is the most amplified mode displayed but the three leading eigenvalues of (4.9) as well.

\section{Results}

\subsection{Optimal body forcing}

Optimal harmonic forcing by means of a distributed body force inside the jet pipe, as outlined in $\S 4.1$, is computed first. The gain (4.2) is displayed in figure $4(a)$ as a function of the Strouhal number. Different line styles represent results obtained for various lengths of the computational domain, in order to assess the influence of domain truncation. Figure 5 displays the spatial distributions of axial velocity of forcing and flow response at selected Strouhal numbers, for a domain length $x_{\max }=40$. It is found from figure 4(a) that domain truncation only affects the gains at very low Strouhal numbers. The flow response structure in this regime extends far downstream, as can be seen in figure $5(a)$, and the truncation at the outflow therefore leads to a lower measure of the flow response norm. Neither the forcing distribution nor the captured part of the flow response appear to be significantly influenced by the downstream truncation. Similarly, it has been verified that a radial truncation at $r=10$ has a negligible impact on the results.

The largest gain is observed at $S t=0.46$. The perturbations in the free jet exhibit a strong spatial growth in the shear-layer just downstream of the nozzle exit; their amplitude peaks near the end of the potential core at $r=8$ (figure $5(b)$ ). In the adjacent decaying part of the wavepacket, the radial amplitude distribution changes markedly, with its maximum now at the centreline. The wavepacket structure at higher frequencies displays similar characteristics, but the region of spatial growth is confined to an ever smaller distance from the nozzle.

The optimal distribution of the body force inside the pipe also exhibits consistent characteristics at all Strouhal numbers presented in figure 5. The amplitude is largest within the boundary layer at the pipe wall, and it is increasingly concentrated near the wall at higher Strouhal numbers. At the same time, the downstream spatial growth of the response increases with the Strouhal number, and its wavelength shortens. In all cases, 
(a)

(b)
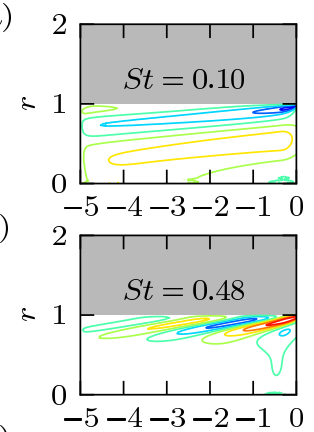

(c)

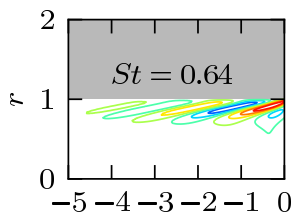

$(d)$

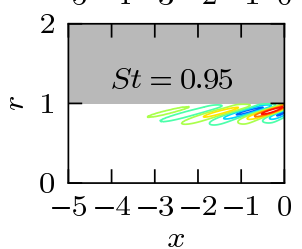

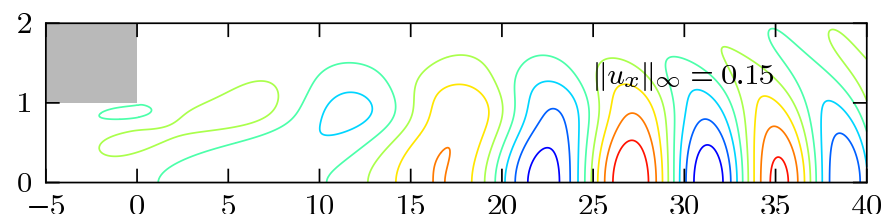
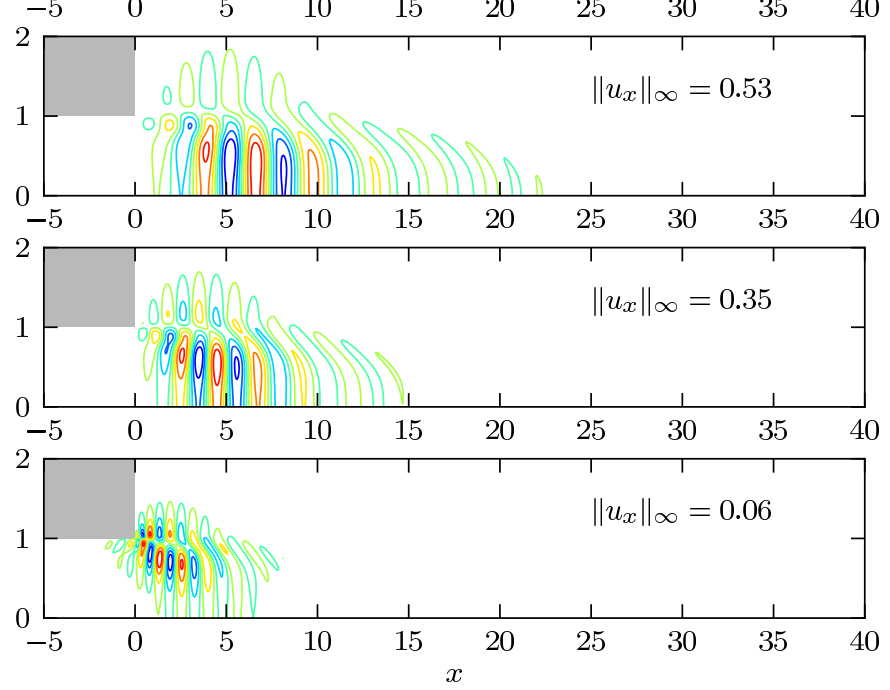

Figure 5. Spatial structures associated with the optimal body forcing at different Strouhal numbers, indicated in the figures. In the left column, the real parts of the axial component of the forcing is displayed. On the right, the figures show the axial component of the response velocity (real part). Computations were performed for $x_{\max }=40$.

the forcing structures are tilted upstream away from the wall, suggesting that the Orr mechanism contributes to the perturbation gain as in the case of boundary layer flow (Sipp \& Marquet 2012).

Use of the $L^{2}$ norm as a measure of the amplification gain inevitably implies that spatially extended structures are given more weight than spatially localized structures, even though the latter may represent modes with high spatial amplification. This effect is undoubtedly responsible for the slight increase of $G_{o p t}^{b f}$ at very low Strouhal numbers. The infinity norm would provide a sensible and intuitive measure for the amplification of perturbations; unfortunately, this norm does not lend itself to the formulation of the optimization problem. It can however be determined a posteriori for the results obtained with the present approach. Values are given in figure 5 for the four cases represented. It is indeed found that the infinity norm follows the same trends as the gain defined by the $L^{2}$ norm, except for the increase at very low Strouhal numbers.

Figure 6(a) displays the maximum amplification curves obtained when the length of pipe included in the computational domain is increased from 5 to 10 . It shows that this parameter affects the values of the gain but that the shape of the curve remains the same. In particular the optimal Strouhal number does not change, which confirms the relevance of the choice of geometric parameters used in this study. A more critical parameter in this analysis is the Reynolds number, as a model turbulent mean flow is used as a base state for the stability analysis so the choice is rather arbitrary: figure $6(b)$ indicates that the 

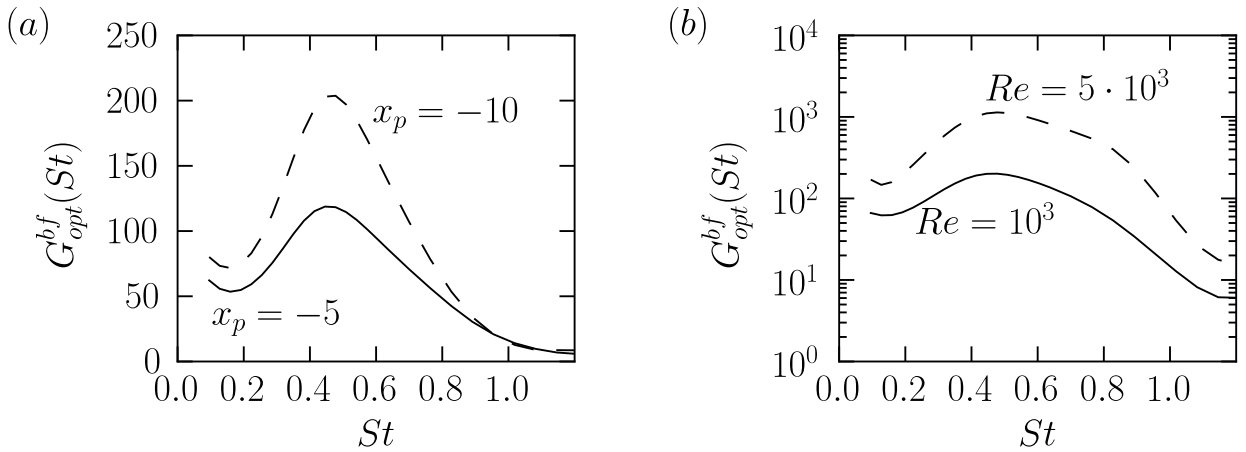

FIGURE 6. Optimal gains obtained for boundary forcing when $(a)$ the length of pipe under included in the computational domain increases from 5 to 10, and $(b)$ the Reynolds number increases from $10^{3}$ to $5 \cdot 10^{3}$.

(a)
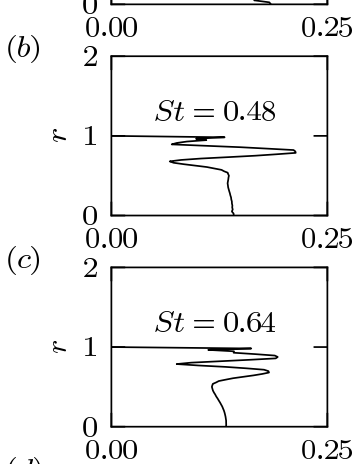

$(d)$

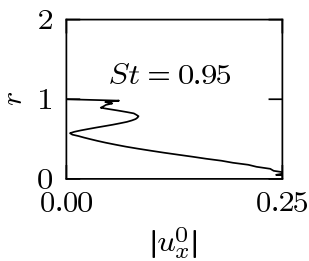

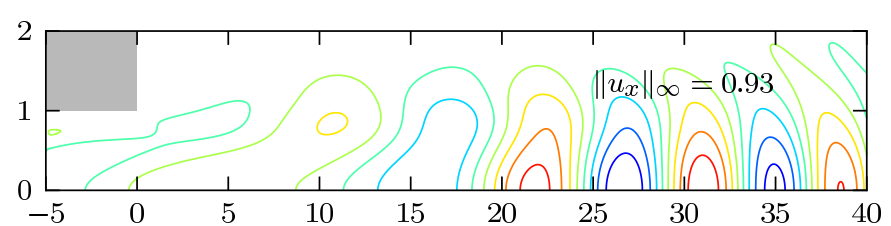
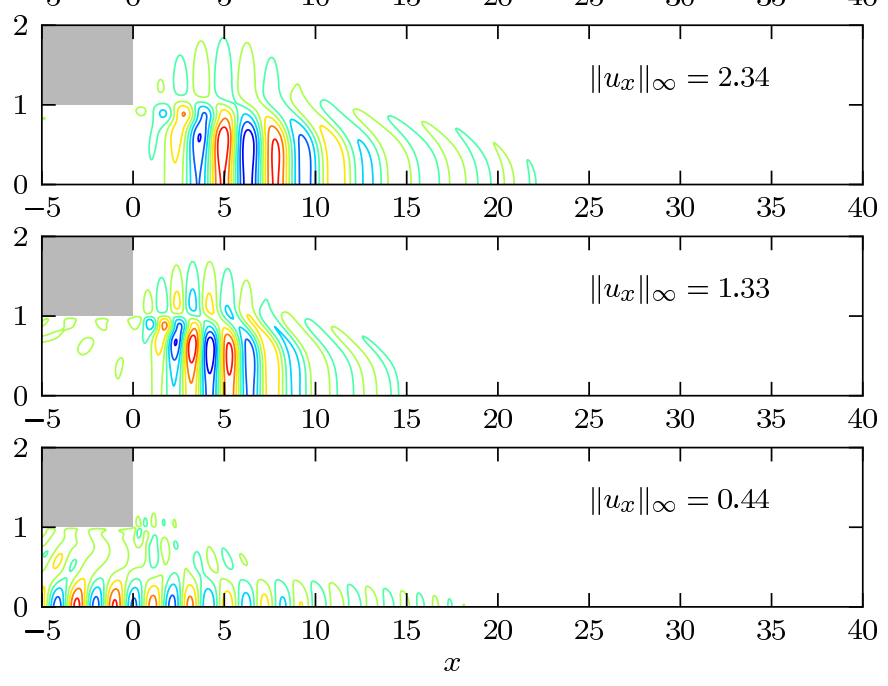

FIGURE 7. Spatial structures associated with the optimal boundary forcing at different Strouhal number, indicated in the figures. In the left column, the modulus of the inflow axial velocity component is displayed. On the right, the axial component of the response velocity is displayed (real part). Computations were performed for $x_{\max }=40$.

optimal excitation frequency remains the same when the Reynolds number is increased from $10^{3}$ to $5 \cdot 10^{3}$. 


\subsection{Optimal boundary forcing}

The perturbation gain obtained from the problem formulation based on forcing at the inflow boundary, as given in $\S 4.2$, is presented in figure $4(b)$. The trends are very similar to those observed in the case of a distributed body force. The strongest amplification occurs at $S t=0.43$. Domain truncation has no influence, except at very low Strouhal numbers, and the results are converged with respect to mesh resolution. The radial distribution of the optimal forcing input is displayed in figure 7 , alongside the flow response at the same four values of $S t$ as in the preceding section. The flow response wavepackets are indeed nearly identical to those of figure 5 , except for the highest Strouhal number shown. The forcing distributions display some unexpected features. At low $S t$, the amplitude maximum is located on the centreline, whereas in the intermediate frequency range the highest forcing amplitudes occur in the pipe boundary layer. The no-slip condition requires the forcing to be zero at $r=1$, but the amplitude is expected to jump to a finite value over a distance of the order of the thickness of the Stokes boundary layer, which scales as $(\omega R e)^{-1 / 2}$ (Batchelor 1967). Both forcing and flow response are of a different character at the highest Strouhal number shown in figure 7 . Perturbations are induced around the centreline; they experience weak growth inside the pipe and immediately decay as they enter the free jet. A closer inspection of the gain curves reveals that the high-St regime is dominated by a formerly sub-optimal branch of singular values. Two additional branches are displayed in figure 4(b). Although barely visible, one of these branches becomes dominant around $S t \approx 1$. The perturbation distribution shown in figure $7(d)$ belongs to this distinct branch.

\subsection{Comparison with local instability results}

The structure of the response wavepackets in figures 5 and 7 is readily understood from well-known local instability characteristics of jet flows (Jendoubi \& Strykowski 1994; Lesshafft 2007). Strong spatial growth takes place in the potential core region, where the shear-layer is thin compared to the instability wavelength. The perturbation amplitude of this local shear-layer mode is concentrated around $r=1$. Downstream of the potential core, the shear-layer mode stabilizes, and the jet-column mode takes over as the least stable, spatial local eigenmode. The amplitude of the jet-column mode in the self-similar base-flow region peaks on the jet axis. The gradual streamwise transition from a shearlayer mode to a jet-column mode is visualized in figure 8 for the wavepacket shown in figure $5(b)$. The thick line represents the local growth rate of the wavepacket, computed as $\frac{1}{2} \partial_{x}(\log E)$ with $E(x)$ as the perturbation kinetic energy at each streamwise station $x$ integrated in the radial direction. The thin solid and dashed lines trace the spatial growth rates of the local shear-layer and jet-column modes, respectively, as functions of $x$. The growth rate of the global wavepacket quickly adapts to that of the shear-layer mode near $x=0$, and it follows its decrease throughout the unstable interval. Downstream of $x \approx 5$, the global wavepacket gradually adjusts to the growth rate of the jet-column mode.

Contrary to what one might initially expect, the forcing structures displayed in figures 5 and 7 bear little resemblance to the local instability modes of the parallel flow inside the pipe. In fact, the optimization algorithm aims at finding the inflow condition that optimally excites shear-layer structures such that the wave packet in the free jet is generated with a maximum amplitude. To this end, the inflow condition consists of a superposition of local instability modes in order to exploit spatial transient amplification mechanisms (Andersson et al. 1999). 


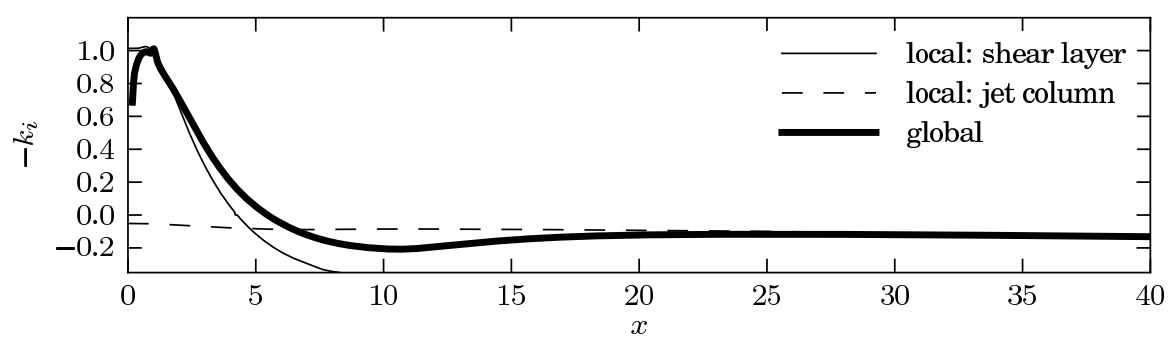

FiguRE 8. Spatial growth rate of the wavepacket envelope (thick line) corresponding to figure $5(b)$, compared to spatial growth rates of the local spatial shear-layer mode (thin line) and the jet-column mode (dashed line) at $S t=0.43$.

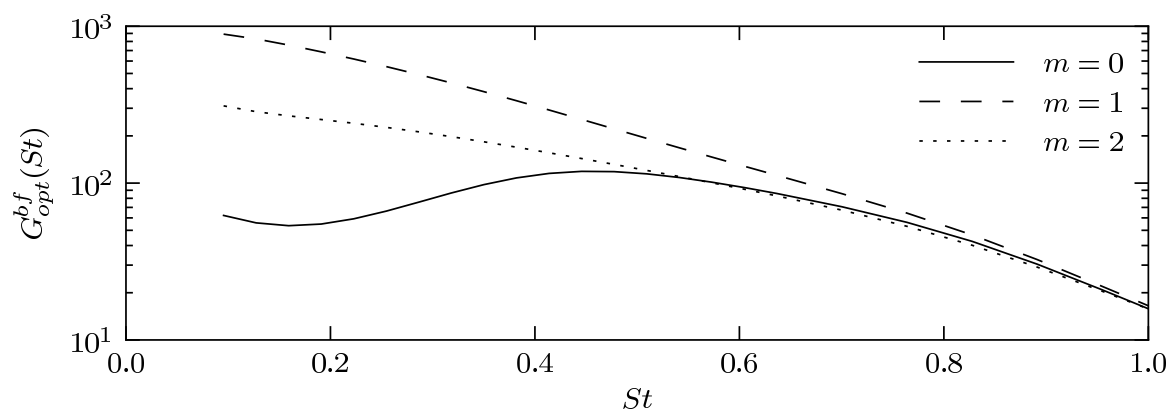

FiguRE 9. Optimal amplification of body forcing for various azimuthal wave-numbers $m$.

\section{Conclusions}

The linear dynamics of forced structures in a jet has been studied within a fully nonparallel framework, so that the effects of the base-flow spreading and of the presence of a solid jet pipe can be taken into account. Unlike approaches using the WKBJ or PSE approximations where the frequency and inflow disturbance profile are imposed to solve for the flow evolution downstream, the present method only seeks the optimal spatial distribution of time-harmonic forcing at a given frequency.

It has been demonstrated that there is no least damped global mode that can resonate in the presence of frequency forcing. The preferred frequency obtained in the present analysis is therefore due to a pseudo-resonance rather than to a resonance as conjectured by Monkewitz (1989) and Huerre \& Monkewitz (1990). The analysis of Cooper \& Crighton (2000) relies on a tangent approximation of the local dispersion relation so as to obtain a "global mode" with a Gaussian envelope. Such an assumption is unlikely to hold in a full WKB approach or in the global analysis followed here. For this reason, one should not expect to recover the "global modes" of Cooper \& Crighton in the present analysis.

Whether external forcing is modeled as an inflow condition or a body force, the amplification of external forcing has been found to be largest for a Strouhal number around 0.45 . This preferred frequency is in good agreement with experimental observation at low forcing intensity (Moore 1977; Crow \& Champagne 1971). Note however that, as shown in the latter reference, the preferred frequency depends on the amplitude of excitation through non-linear effects. Around this optimal frequency, the excitation generates a 
wavepacket that develops in the free-jet shear-layer. It is amplified through the potential core, where shear is important, and decays further downstream while it gets localized on the centreline. This behaviour is consistent with local stability results that show that, while shear-layer modes are spatially unstable in the potential core, the jet column mode becomes the least damped spatial eigenmode further downstream.

The shape of the optimal body forcing indicates that the Orr mechanism is at play to generate perturbations that grow in the jet pipe boundary layer and then optimally excite the free-jet wavepacket. The results are not very sensitive to the actual shape of the forcing term as similar results are obtained for body and boundary forcing. In both cases, a good agreement is found between the most amplified wavepacket and the experimentally observed preferred mode.

The framework of optimal forcing is therefore a suitable tool for the analysis of the non-modal instabilities developing in convection dominated amplifier flows.

Local spatial stability analysis indicates that helical perturbations, unlike axisymmetric ones, are spatially amplified downstream of the potential core, as shown e.g. by Batchelor \& Gill (1962) and Michalke (1984). This is especially true in the low frequency range. Computations have been performed using the current framework for higher azimuthal wave numbers $m$. For $m \neq 0, G_{o p t}(S t)$ is a monotonically decreasing function of $S t$, and the levels obtained at low frequencies are indeed larger for $m=1,2$ than for $m=0$ (see figure 9). However this is not only due to a faster growth of the wave packet downstream of the nozzle, but also and most importantly to a spatial amplification over a longer streamwise distance, resulting in larger $L^{2}$ norms for the flow response. The growth of the wavepacket through the potential core is however similar for all values of $m$.

Experiments typically do not show a dominance of $m=1$ helical modes in the selfsimilar region. Several reasons may explain this discrepancy between the results in figure 9 and observations, in particular the effects of turbulence and nonlinear saturation, which are not captured in the present analysis. In this light, the $L^{\infty}$ norm might provide a more relevant and intuitive measure of the perturbation amplification. The use of such a formulation will be explored in future studies.

This work was supported by DGA grant number 2009.60.034.00.470.75.01 and by a fellowship from the EADS Foundation. Computational resources were provided by GENCI (Grant 2012-026451).

\section{REFERENCES}

Alizard, F., Cherubini, S. \& Robinet, J.-C. 2009 Sensitivity and optimal forcing response in separated boundary layer flows. Physics of Fluids 21 (6), 064108.

Andersson, P., Berggren, M. \& Henningson, D. S. 1999 Optimal disturbances and bypass transition in boundary layers. Physics of Fluids 11 (1), 134.

Balay, S., Buschelman, K., Eijkhout, V., Gropp, W. D., Kaushik, D., Knepley, M. G., McInnes, L. Curfman, Smith, B. F. \& Zhang, H. 2008 PETSc users manual. Tech. Rep. ANL-95/11 - Revision 3.3.0. Argonne National Laboratory, available at http://www.mcs.anl.gov/petsc/petsc-as/.

BARKLEY, D 2006 Linear analysis of the cylinder wake mean flow. Europhysics Letters 75 (5), $750-756$.

BAtChelor, G. K. 1967 An introduction to fluid dynamics. Cambridge: Cambridge Univ. Press.

Batchelor, G. K. \& Gill, A. E. 1962 Analysis of the stability of axisymmetric jets. Journal of Fluid Mechanics 14 (04), 529.

Cooper, A. J. \& Crighton, D. G. 2000 Global modes and superdirective acoustic radiation in low-speed axisymmetric jets. European Journal of Mechanics - B/Fluids 19 (5), 559-574. 
Crighton, D. G. \& Gaster, M. 1976 Stability of slowly diverging jet flow. Journal of Fluid Mechanics 77 (02), 397.

Crow, S. C. \& Champagne, F. H. 1971 Orderly structure in jet turbulence. Journal of Fluid Mechanics 48 (03), 547.

Dick, E. 2009 Introduction to finite element methods in computational fluid dynamics. In Computational fluid dynamics: an introduction, 3rd edn. Springer.

Garnaud, X. 2012 Modes, transient dynamics and forced response of circular jets. PhD thesis, Ecole Polytechnique.

Garnaud, X., Lesshafft, L. \& Huerre, P 2011 Global linear stability of a model subsonic jet. AIAA paper 2011-3608.

Gudmundsson, K. \& Colonius, T. 2011 Instability wave models for the near-field fluctuations of turbulent jets. Journal of Fluid Mechanics 689, 97-128.

Hеснт, F. 2011 Freefem ++ manual, third edition, version 3.2. Tech. Rep.. Available at http://www.freefem.org/ff++.

Hernandez, V., Roman, J. E. \& Vidal, V. 2005 SLEPc: a scalable and flexible toolkit for the solution of eigenvalue problems. ACM Transactions on Mathematical Software 31 (3), 351362 .

Huerre, P. \& Monkewitz, P. A. 1990 Local and global instabilities in spatially developing flows. Annual Review of Fluid Mechanics 22, 473-537.

Jendoubi, S. \& Strykowski, P. J. 1994 Absolute and convective instability of axisymmetric jets with external flow. Physics of Fluids 6 (9), 3000.

LesshaffT, L. 2007 Global modes and aerodynamic sound radiation in self-excited hot jets. $\mathrm{PhD}$ thesis, Ecole Polytechnique.

Marquet, Olivier \& Sipp, Denis 2010 Global sustained perturbations in a backward-facing step flow. In Seventh IUTAM Symposium on Laminar-Turbulent Transition (ed. Philipp Schlatter \& Dan S. Henningson), IUTAM Bookseries, vol. 18, pp. 525-528. Springer Netherlands.

Matsushima, T. \& Marcus, P. S. 1995 A spectral method for polar coordinates. Journal of Computational Physics 120, 365-374.

Michalke, A. 1984 Survey on jet instability theory. Progress in Aerospace Science 21, 159-199.

Monkewitz, P. A. 1989 Feedback control of global oscillations in fluid systems. AIAA Paper 89-0991.

Monkewitz, P. A. \& Sohn, K. 1988 Absolute instability in hot jets. AIAA Journal 26 (8), 911-916.

Monokrousos, A., Akervik, E., Brandt, L. \& Henningson, D. S. 2010 Global threedimensional optimal disturbances in the blasius boundary-layer flow using time-steppers. Journal of Fluid Mechanics 650, 181.

Moore, C. J. 1977 The role of shear-layer instability waves in jet exhaust noise. Journal of Fluid Mechanics 80 (02), 321.

Nichols, J. W. \& LeLE, S. K. 2010 Global mode analysis of turbulent high-speed jets. Annual research briefs 2010. Center for Turbulence Research.

Nichols, J. W. \& LeLE, S. K. $2011 a$ Global modes and transient response of a cold supersonic jet. Journal of Fluid Mechanics 669, 225-241.

Nichols, J. W. \& LeLE, S. K. $2011 b$ Non-normal global modes of high-speed jets. International Journal of Spray and Combustion Dynamics 3 (4), 285-302.

PIER, B. 2002 On the frequency selection of finite-amplitude vortex shedding in the cylinder wake. Journal of Fluid Mechanics 458.

Ray, P. K., Cheung, L. C. \& Lele, S. K. 2009 On the growth and propagation of linear instability waves in compressible turbulent jets. Physics of Fluids 21 (5), 054106.

Rodriguez, D., Samanta, A., Cavalieri, A.V.G, Colonius, T. \& Jordan, P. 2011 Parabolized stability equation models for predicting large-scale mixing noise of turbulent round jets. In Proceedings of the 17th AIAA/CEAS Aeroacoustics Conference. Portland, Oregon.

Sipp, Denis \& Marquet, Olivier 2012 Characterization of noise amplifiers with global singular modes: the case of the leading-edge flat-plate boundary layer. Theoretical and Computational Fluid Dynamics pp. 1-19.

Trefethen, L. N., Trefethen, A. E., Reddy, S. C. \& Driscoll, T. A. 1993 Hydrodynamic stability without eigenvalues. Science 261 (5121), 578-584. 